\title{
A review of ropinirole prolonged release in Parkinson's disease
}

\author{
Muhammad M Nashatizadeh \\ Kelly E Lyons \\ Rajesh Pahwa
}

Department of Neurology, University of Kansas Medical Center, Kansas City, Kansas, USA

\begin{abstract}
Ropinirole prolonged release is a once-daily, 24-hour formulation of ropinirole, a non-ergot dopamine agonist. It is approved as monotherapy and as an adjunct to levodopa in the treatment of Parkinson's disease (PD). Several potential advantages of ropinirole prolonged release compared to the immediate release formulation include maintaining more consistent dopaminergic activity with steadier plasma levels, increased tolerability, greater compliance from a simpler once-daily dosing regimen and ease in dose titration. In a randomized, double-blind, non-inferiority, crossover study, ropinirole prolonged release was shown to have comparable efficacy and tolerability to immediate release ropinirole in early PD patients, with significantly greater compliance. Subjects were converted overnight between ropinirole formulations without loss of efficacy and with good tolerability. In a randomized, double-blind, placebo-controlled study in advanced PD, daily "off" time was reduced by an average of 2.1 hours with ropinirole prolonged release compared to 0.4 hours with placebo. Patients on ropinirole prolonged release were also more likely to require less daily levodopa. Ropinirole prolonged release is well tolerated with a similar adverse effect profile to other non-ergot dopamine agonists. The most common adverse effects include dyskinesia, nausea, dizziness, hallucinations, somnolence, abdominal pain or discomfort and orthostatic hypotension. Ropinirole prolonged release is a safe and effective treatment option for both early and advanced PD. This manuscript briefly reviews the current pharmacological treatment options for PD and provides a more detailed review of the currently available data regarding ropinirole prolonged release as a treatment option for PD.
\end{abstract}

Keywords: ropinirole prolonged release, ropinirole PR, dopamine agonist, Parkinson's disease, once-daily treatment

\section{Introduction}

Parkinson's disease (PD) is a progressive neurological disorder with the primary symptoms of bradykinesia, tremor, and rigidity as well as postural instability as the disease advances. Although the average age of onset is 60 years, adults of all ages can be diagnosed with PD. It is estimated that the prevalence of PD in the United States (US) is approximately one million persons ${ }^{1}$ and approximately 50,000 new cases are diagnosed each year. ${ }^{2}$ The lifetime risk of developing PD is estimated to be $2.0 \%$ for men and $1.3 \%$ for women. ${ }^{3}$

At the time of diagnosis, the initiation of treatment is based on several factors such as age, other medical conditions, cognitive and psychiatric status, employment, social situation and severity of symptoms. Treatment is generally initiated when the symptoms of PD are bothersome to the patient or interfering with the patient's daily functioning. There are currently several treatment options available for PD that can lessen physical disability, reduce signs and symptoms of the disease and improve overall quality of life..$^{4-7}$ These treatments include carbidopa/levodopa, monoamine oxidase type $\mathrm{B}$ (MAO-B) inhibitors, dopamine agonists, catechol- $O$-methyltransferase (COMT) inhibitors, anticholinergics, and amantadine (Table 1). This review will briefly discuss the treatment options available for PD and will focus on ropinirole prolonged 
Table I Available pharmacological treatment options for Parkinson's disease

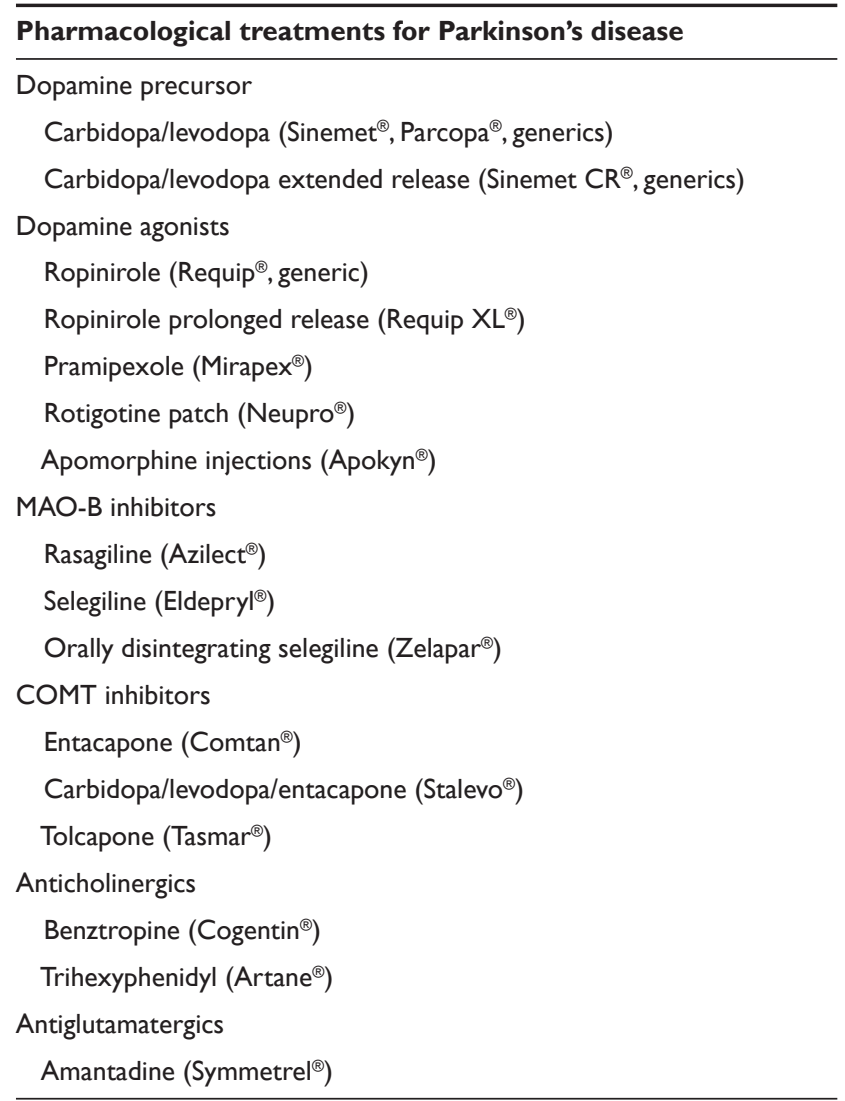

Abbreviations: COMT, catechol-O-methyltransferase; MAO-B, monoamine oxidase type B.

release, a once-daily non-ergot dopamine agonist approved by the US Food and Drug Administration (FDA) in 2008.

\section{Treatment of early Parkinson's disease}

Carbidopa/levodopa is the most effective treatment option for PD and at some point during the disease course will be required by almost all PD patients. ${ }^{8}$ However, even early in the disease course, carbidopa/levodopa can lead to the development of motor complications such as motor fluctuations and dyskinesia. ${ }^{9}$ Motor fluctuations generally initially present as a re-emergence of PD symptoms at the end of a given dose, prior to the scheduled intake of the next dose often referred to as end of dose wearing off. As the disease progresses, "on/off" fluctuations may occur in which the patient fluctuates between "on" periods when the medication is controlling PD symptoms and "off" periods when symptoms are not controlled. Initially these fluctuations tend to be predictable and progress to unpredictable fluctuations between the "on" and "off" states. It has been suggested that motor fluctuations may be a result of pulsatile stimulation of dopamine receptors due to the short duration response of multiple daily doses of oral levodopa. ${ }^{10}$ Dyskinesia are involuntary wiggling or dance-like movements that most commonly occur at peak levodopa blood concentrations. ${ }^{8}$ Risk factors for the development of levodopa-induced motor complications include longer disease duration, longer duration of levodopa therapy, higher doses of levodopa, often over $300 \mathrm{mg} /$ day, ${ }^{9}$ greater disease severity and younger age of disease onset. ${ }^{11}$ More specifically, it has been demonstrated that a higher daily levodopa dose per kilogram body weight may be a stronger predictor of the development of dyskinesia than the absolute amount of daily levodopa. ${ }^{12}$ Given the potential for levodopa to cause motor complications, it is a common treatment strategy to initiate therapy with an alternate medication and add levodopa later in the disease course as symptoms become more severe. ${ }^{8} \mathrm{MAO}-\mathrm{B}$ inhibitors and dopamine agonists are commonly used as initial treatment options for PD. ${ }^{4,6}$

MAO-B metabolizes dopamine, therefore, MAO-B inhibitors, selegiline and rasagiline, block the MAO-B enzyme resulting in reduced metabolism of dopamine and hence more dopamine in the brain. Although only approved as an adjunctive treatment to levodopa in the US, selegiline, at doses of $5 \mathrm{mg}$ in the morning and afternoon, has been shown to have mild benefit in the treatment of early PD. ${ }^{4}$ Selegiline is metabolized to amphetamine and methamphetamine metabolites which can lead to adverse effects such as confusion and hallucinations. Rasagiline is approved for the treatment of early PD at a dose of $1 \mathrm{mg}$ taken once daily and results in a mild improvement in PD symptoms. It is metabolized to aminoindan rather than amphetamine and methamphetamine metabolites and therefore does not have the related side effects seen with selegiline and overall has minimal adverse effects. ${ }^{6}$ In addition, in a delayed start study design with rasagiline it was reported that patients who started rasagiline earlier had better Unified Parkinson's Disease Rating Scale (UPDRS) scores at one year compared to patients who initiated with placebo and started rasagiline 6 months later. ${ }^{13}$

Dopamine agonists directly stimulate the post-synaptic dopamine receptors. The non-ergot dopamine agonists, ropinirole and pramipexole, are currently the most commonly used. They have both been shown to be efficacious in the treatment of early PD and to delay the development of motor complications when compared to levodopa. ${ }^{14,15}$ Pramipexole is generally dosed three times daily and slowly titrated over several weeks to a maximum daily dosage of $4.5 \mathrm{mg}$. 
Ropinirole is also generally given three times daily and slowly titrated over several weeks to a maximum dose of $24 \mathrm{mg}$. In general dopamine agonists are considered to be less efficacious than levodopa. ${ }^{6}$ Dopamine agonists have an increased risk of adverse effects compared to levodopa, namely, somnolence, hallucinations, edema and impulse control disorders. ${ }^{4,16}$ Rotigotine is a dopamine agonist formulated as a continuous release transdermal patch approved for the treatment of early PD in the US at doses up to $6 \mathrm{mg} / 24$ hours; however, it was recalled by the FDA in 2008 after crystallization of the patch release system led to unpredictable drug release and variable plasma drug levels. ${ }^{17}$ Rotigotine is available in countries outside the US at daily dosages up to $8 \mathrm{mg} / 24$ hours for early PD. The older, ergot-derived dopamine agonists, bromocriptine and pergolide as well as cabergoline which is not approved as a treatment for PD in the US, have been associated with an increased risk of cardiac valvular disease, pleuropulmonary fibrosis and pleural effusion thereby limiting their use. ${ }^{18,19}$ In fact, pergolide was recalled by the FDA in 2007 after 19 years on the US market citing these safety concerns.

Rarely, anticholinergics, most commonly benztropine and trihexyphenidyl, are used in the treatment of early PD. Anticholinergics have been shown to have a mild effect on PD symptoms, primarily tremor. ${ }^{4}$ Benztropine is initiated at $0.5 \mathrm{mg}$ twice daily and increased by 0.5 to $1.0 \mathrm{mg}$ every 3 to 4 days as necessary. Trihexyphenidyl is generally initiated at $1 \mathrm{mg}$ once or twice daily and increased by 0.5 to $1.0 \mathrm{mg}$ every 3 to 4 days as necessary. Amantadine has also been shown to have a modest effect in early PD. ${ }^{4}$ It is a glutamateric N-methyl-D-aspartate (NMDA) antagonist and has been postulated to increase dopamine release, decrease the reuptake of dopamine and stimulate dopamine receptors although the exact mechanism of action in PD is not entirely clear. Amantadine is generally initiated at $100 \mathrm{mg} /$ day and increased up to $300 \mathrm{mg} /$ day as necessary. Although higher doses can be used, it is not recommended due to an increase in adverse effects such as confusion and hallucinations. ${ }^{8}$

\section{Treatment of advanced Parkinson's disease}

There are multiple treatment options available for advanced PD patients that develop levodopa-induced motor fluctuations and dyskinesia. ${ }^{7,8}$ One approach is to adjust the dosage or dosing frequency of levodopa or to switch to an extended release formulation of levodopa. However, several studies have demonstrated that the extended release formulation of levodopa does not reduce "off" time compared to the immediate release formulation. ${ }^{7}$ Another approach is to add an adjunctive therapy to levodopa such as amantadine, an MAO-B inhibitor, a COMT inhibitor, and/or a dopamine agonist.

Amantadine has been shown to be effective in reducing dyskinesia. ${ }^{7}$ In a double-blind study, amantadine $(100 \mathrm{mg}$ twice daily) was shown to reduce dyskinesia by $24 \%{ }^{20}$ Although not available in the US, an intravenous formulation of amantadine (amantadine sulfate) has been shown to reduce dyskinesia by up to $50 \%$ after an infusion of $200 \mathrm{mg}$ compared to placebo. ${ }^{21}$

The MAO-B inhibitors, selegiline, orally disintegrating selegiline and rasagiline are approved as adjunct treatments to levodopa. Selegiline is generally given twice daily with $5 \mathrm{mg}$ taken in the morning and afternoon. There are insufficient data on the effects of selegiline on motor complications and it has been concluded that it may possibly reduce motor fluctuations. ${ }^{7}$ Orally disintegrating selegiline is a newer formulation of selegiline which has fewer amphetamine metabolites and eliminates the need for swallowing pills. It is approved for the treatment of patients with levodopa-induced wearing off and has been shown to reduce "off" time by 2.2 hours per day compared to 0.6 hours with placebo. ${ }^{22}$ Orally disintegrating selegiline is initiated at $1.25 \mathrm{mg}$ once daily and increased after 6 weeks if needed to $2.5 \mathrm{mg}$ once daily. The American Academy of Neurology (AAN) evidence-based review of treatment options for motor fluctuations concluded that rasagiline was effective in reducing "off" time and should be offered as a treatment option. Reductions in daily "off" time ranged between 0.5 and 1.0 hours per day depending on the dose of rasagiline. ${ }^{7}$ In advanced PD, rasagiline is initiated at $0.5 \mathrm{mg}$ once daily and increased to $1.0 \mathrm{mg}$ once daily as needed.

COMT inhibitors are another adjunctive option for the treatment of advanced PD. The enzyme COMT breaks down levodopa; therefore, the addition of a COMT inhibitor allows more levodopa to reach the brain. Entacapone and tolcapone are the COMT inhibitors available for the treatment of advanced PD and they are used only in conjunction with levodopa. The AAN evidence-based guidelines concluded that entacapone is effective in reducing "off" time an average of 0.7 to 1.2 hours per day and should be offered to patients with motor fluctuations. ${ }^{7}$ Entacapone is available in $200 \mathrm{mg}$ tablets. One tablet is given with each dose of levodopa, not to exceed a total daily dose of $1600 \mathrm{mg}$ of entacapone. Entacapone is also available in a triple combination tablet containing carbidopa, levodopa and entacapone. There are six strengths of the triple combination available in which each 
tablet contains $200 \mathrm{mg}$ of entacapone and either 50, 75, 100, 125,150 or $200 \mathrm{mg}$ of levodopa and carbidopa equivalent to one quarter of the levodopa dose. Tolcapone has been determined to be probably effective in the treatment of "off" time with an average daily reduction in "off" time of 0.9 to 1.8 hours. $^{7}$ It was also noted that tolcapone should be used with caution due to potential hepatotoxicity and requires regular safety monitoring. Tolcapone is initiated at $100 \mathrm{mg}$ three times daily and increased to $200 \mathrm{mg}$ three times daily if necessary. If there is no reduction in "off” time after 3 weeks of tolcapone therapy it should be discontinued.

The dopamine agonists pramipexole and ropinirole have been determined to probably be effective in reducing "off" time and should be considered as a treatment option. ${ }^{7}$ Pramipexole has been shown to reduce "off" time by an average of $12 \%$ to $24 \%$ and ropinirole by an average of $7 \%$ to $19 \%$. Both medications are generally given three times daily for advanced PD with a maximum daily dose of $4.5 \mathrm{mg}$ of pramipexole and $24 \mathrm{mg}$ of ropinirole. The rotigotine patch is not approved for advanced PD in the US; however, outside of the US it is approved at dosages up $16 \mathrm{mg} / 24$ hours. Rotigotine $8 \mathrm{mg} / 24$ hours was shown to reduce "off" time by 1.8 hours a day and the $12 \mathrm{mg} / 24$ hour dose by 1.2 hours a day. ${ }^{23}$ Cabergoline, a once-daily, ergot dopamine agonist, has been shown to reduce "off" time by $18 \%$. $^{7}$ Apomorphine is the only injectable medication approved for the treatment of PD. It is a fast-acting, short-duration treatment used for advanced PD as a rescue therapy for patients with severe "off" periods and has been shown to reduce daily "off" time by an average of 2 hours. ${ }^{24}$

A common problem in early and advanced PD is patient medication compliance. In early disease, patients generally initially receive benefit from their treatment regimen in spite of late or missed doses of medication. As the disease progresses, multiple medications are often prescribed with more complicated dosing schedules and in order to maintain benefit and avoid motor complications, adherence to the prescribed dosing schedule becomes an important issue. In a 4-week study of medication compliance in PD patients with a dosing schedule requiring the intake of medication at least three times daily, only $10 \%$ of the patients had complete compliance. Furthermore, it was reported that $51 \%$ of the patients missed at least one dose a week, $21 \%$ missed at least three doses per week and $82 \%$ of patients did not take their medication on time. ${ }^{25}$ The most common reasons for non-compliance were forgetting to take their medication or not having access to their medication at the scheduled dosing time. Medication compliance was also measured in a larger multicenter study of 112 PD patients using electronic monitoring bottles. ${ }^{26}$ The median overall compliance for taking the amount of medication prescribed was $97.7 \%$, compliance for taking the medication on the right day was $86.2 \%$ and compliance for taking it at the right time was $24.4 \%$. It was also shown that those with suboptimal compliance had worse motor scores and those taking once-daily preparations had better compliance than those taking multiple daily doses. This study highlights the need for medications with less complicated dosing schedules with fewer daily doses. In 2008, the FDA approved ropinirole prolonged release which is a once daily 24-hour formulation of the dopamine agonist, ropinirole. This is the first once-daily oral dopamine agonist approved for both early and advanced PD in the US.

\section{Ropinirole}

Ropinirole is the active drug in ropinirole prolonged release. Ropinirole is a non-ergot D2/D3 dopamine agonist with the greatest affinity at the D3 receptors. ${ }^{27}$ It has affinity to central and peripheral dopamine receptors. Ropinirole is absorbed rapidly with peak plasma concentrations occurring in 1 to 2 hours. After 2 days of use, steady state concentrations are generally achieved. The bioavailability is approximately $55 \%$ but absorption may be delayed by an average of 2 hours after consuming a meal high in fat compared with the fasting state. Nevertheless, no clinically significant differences in $\mathrm{C}_{\min }, \mathrm{C}_{\max }$ or $\mathrm{AUC}_{24}$ between fasting and fed states have been noted. ${ }^{27}$

Since the pharmacokinetics of ropinirole have not been studied in patients with hepatic impairment it should be titrated with caution in patients with hepatic insufficiency. No specific modifications in dosing are necessary in patients with mild to moderate renal insufficiency as characterized by a creatine clearance of 30 to $50 \mathrm{~mL} / \mathrm{min}$ because only $10 \%$ of the drug is excreted unchanged in urine. Ropinirole has not been studied in patients with severe renal impairment. Plasma protein binding is highly variable between $10 \%$ and $40 \%$ with an overall volume of distribution of about $7.5 \mathrm{~L} / \mathrm{kg} .{ }^{28}$ The elimination half-life of ropinirole is about 6 hours and this is mainly through cytochrome $\mathrm{P}_{450}$ metabolism by isozyme CYP1A2 and to a lesser extent by isozyme CYP3A. ${ }^{27}$

\section{Potential interactions and special populations}

Ciprofloxacin is a second generation fluoroquinolone antibiotic and known inhibitor of CYP1A2 that was found to increase ropinirole $\mathrm{C}_{\max }$ by about $60 \%$ and AUC by $84 \%$. Other clinically relevant potential CYP1A2 inhibitors include 
amiodarone, cimetidine, fluvoxamine, interferon, mexilitine and ticlopidine. Patients taking ropinirole may require dosing adjustments with the introduction, withdrawal or dose adjustment in any of these medications. ${ }^{27}$

Conversely, smoking cigarettes is known to induce CYP1A2 thereby reducing $\mathrm{C}_{\max }$ levels by about $30 \%$ in comparison with non-smokers. High-dose estrogen appears to reduce drug clearance by about $35 \%$, so patients who stop taking hormonal replacement therapy might require higher doses of ropinirole to achieve the same clinical effect. ${ }^{27}$ Because ropinirole is a dopamine agonist, the concurrent use of dopamine antagonists such as neuroleptic medications is likely to be counterproductive and diminish the efficacy of either type of medication. Ropinirole itself does not induce or inhibit the cytochrome $\mathrm{P}_{450}$ system, so no significant drug-drug interactions have been noted or are expected with other CYP1A2 substrates. ${ }^{27}$

Given that a large number of patients with $\mathrm{PD}$ who are over the age of 65 , it is worth noting that the drug is cleared $15 \%$ less in older patients. ${ }^{27}$ However, individual dosing titrated to effect makes this population comparison between younger and older patients less clinically meaningful. Many older patients are sensitive to new medications due to physiologic differences so special care should be taken to initiate at the lowest dose and escalate slowly to reduce adverse effects.

\section{Ropinirole prolonged release}

Ropinirole prolonged release is designed as a three-layer tablet with the innermost layer containing ropinirole and two outer barrier layers containing placebo that help to control the amount of surface area available for drug release. ${ }^{29}$ The bioavailability of ropinirole prolonged release compared to the immediate release formulation is nearly $100 \%$. After approximately 4 days of use, steady state concentrations are generally achieved. As would be expected, from a once-daily $8 \mathrm{mg}$ dose of ropinirole prolonged release, the median time to achieve maximum plasma concentration is longer than that of the immediate release formulation dosed as $2.5 \mathrm{mg}$ three times daily ( $\sim 6$ hours vs $\sim 2$ hours $).{ }^{29}$ However, similar steadystate calculations of the area under the curve for a 24-hour period $\left(\mathrm{AUC}_{24}\right)$ were noted between prolonged release and immediate release formulations $(16.3 \mathrm{ng} \times \mathrm{h} / \mathrm{mL} / \mathrm{mg}$ vs $16.0 \mathrm{ng} \times \mathrm{h} / \mathrm{mL} / \mathrm{mg}){ }^{30}$ In addition, similar dose-normalized minimum plasma levels were noted between the two formulations $(0.44 \mathrm{ng} / \mathrm{mL} / \mathrm{mg}$ vs $0.45 \mathrm{ng} / \mathrm{mL} / \mathrm{mg})$. The dosenormalized $\mathrm{C}_{\max }$ for ropinirole prolonged release is slightly lower than the immediate release formulation $(0.92 \mathrm{ng} / \mathrm{mL} / \mathrm{mg}$ vs $1.05 \mathrm{ng} / \mathrm{mL} / \mathrm{mg}$ ), most likely resulting from a more gradual approximation of peak dose. ${ }^{28}$
Ropinirole prolonged release is currently available in the US in $2 \mathrm{mg}, 4 \mathrm{mg}, 8 \mathrm{mg}$, and $12 \mathrm{mg}$ tablets whereas ropinirole immediate release is available in $0.25 \mathrm{mg}, 0.5 \mathrm{mg}$, $1 \mathrm{mg}, 2 \mathrm{mg}, 3 \mathrm{mg}, 4 \mathrm{mg}$ and $5 \mathrm{mg}$ tablets. ${ }^{27,29}$ When switching patients from ropinirole immediate release to ropinirole prolonged release, it is important to consider the total daily dose of immediate release because it is usually administered multiple times daily. For example, a ropinirole immediate release regimen of $4 \mathrm{mg}$ three times a day would equal a total daily dose of $12 \mathrm{mg}$, which is equivalent to a single $12 \mathrm{mg}$ dose of ropinirole prolonged release. Table 2 compares equivalent dosing amounts based on the immediate release total daily dose. ${ }^{29}$ Because ropinirole immediate release is available in doses less than $1 \mathrm{mg}$ ( 0.25 and $0.50 \mathrm{mg})$, conversion to prolonged release doses may approximate but may not always equal 1:1 dosing. For example, a patient taking immediate release $0.75 \mathrm{mg}$ three times a day (total daily dose $=2.25 \mathrm{mg}$ ) would be recommended to switch to prolonged release $2 \mathrm{mg}$ tablet once daily. The prolonged release tablets should never be broken into halves because doing so would break down the process of gradual drug release resulting in supratherapeutic and potentially harmful plasma drug levels. Ropinirole prolonged release is initiated at $2 \mathrm{mg}$ once daily and increased every 1 to 2 weeks by $2 \mathrm{mg}$ until a therapeutic dose is reached or a maximum of $24 \mathrm{mg} /$ day is achieved.

\section{Efficacy studies}

\section{Early Parkinson's disease}

There are currently limited studies of ropinirole prolonged release in the literature. The EASE-PD Monotherapy study was a multicenter, randomized, double-blinded, crossover study involving ropinirole immediate release and ropinirole prolonged release to determine non-inferiority. Participants were required to be within Hoehn and Yahr stage I-III and

Table 2 Conversion from ropinirole immediate release to ropinirole prolonged release $^{29}$

\begin{tabular}{ll}
\hline $\begin{array}{l}\text { Ropinirole immediate release } \\
\text { total daily dose }(\mathbf{m g})\end{array}$ & $\begin{array}{l}\text { Ropinirole prolonged } \\
\text { release daily dose }(\mathbf{m g})\end{array}$ \\
\hline $0.75-2.25$ & 2 \\
$3-4.5$ & 4 \\
6 & 6 \\
$7.5-9$ & 8 \\
12 & 12 \\
$15-18$ & 16 \\
21 & 20 \\
24 & 24 \\
\hline
\end{tabular}


not taking levodopa or dopamine agonists for at least two weeks prior to the study. ${ }^{31}$ The only concomitant antiparkinsonian medications allowed were stable doses of selegiline, amantadine and anticholinergics. After taking placebo for 7 days, 161 patients were randomized into four possible permutations of formulation sequence: 1) immediate release - immediate release - prolonged release, 2) immediate release - prolonged release - prolonged release, 3 ) prolonged release - prolonged release - immediate release and 4) prolonged release - immediate release - immediate release. During the first 12-week period, patients were given the first formulation in their sequence such that $50 \%$ initially received ropinirole immediate release $0.25 \mathrm{mg}$ three times daily and the other $50 \%$ initially received ropinirole prolonged release $2 \mathrm{mg}$ once daily. Over those 12 weeks, patient doses were increased and titrated clinically to achieve efficacy and tolerability up to a maximum of $24 \mathrm{mg}$ daily in either formulation. ${ }^{31}$

After the initial titration period, subjects taking either immediate (8.9 points) or prolonged release (10.4 points) formulations had overall improvement in their UPDRS motor scores but there was no significant difference in efficacy between the two formulations. Only patients who achieved a stable UPDRS motor score over a 2-week period were allowed to proceed to an 8 -week maintenance period. ${ }^{31}$ It is important to note that at the end of these 8 weeks, half of the patients initially taking ropinirole immediate release were switched overnight to the closest available ropinirole prolonged release dose. Likewise, half of the patients initially taking ropinirole prolonged release were switched overnight to the closest available ropinirole immediate release dosing regimen. The remaining patients underwent a dummy switch overnight and therefore remained on the same medication they were already taking. After a second 8-week maintenance period, a similar switch took place whereby this time anyone who had actually switched drugs previously was given a dummy switch overnight, and those who had the dummy switch previously changed to the alternate formulation of drug. In this way, by the end of the second 8-week maintenance period, all participants had successfully been switched to the alternate formulation $(50 \%$ after the first maintenance period and the other $50 \%$ after the second period).

A total of 114 subjects completed the study. ${ }^{31}$ Subjects initially titrated on ropinirole prolonged release were able to begin with a higher total daily dose ( $2 \mathrm{mg}$ instead of $0.75 \mathrm{mg}$ ) and achieve the maximum daily dose of $24 \mathrm{mg}$ with fewer dose titrations (minimum 8 dose titrations) compared to those on immediate release (minimum 13 dose titrations). Only five patients taking ropinirole immediate release required the next higher dose of ropinirole prolonged release after being switched to maintain efficacy. Similarly, only two patients taking ropinirole prolonged release required the next higher dose of ropinirole immediate release after switching. No dose reductions were performed because of adverse effects. The overall mean change in UPDRS motor scores was a clinically insignificant worsening of 0.6 points in the ropinirole immediate release group and a clinically insignificant improvement of 0.1 points for the ropinirole prolonged release group noted throughout the three 8-week maintenance periods suggesting that similar doses have similar efficacy. ${ }^{31}$ Inferiority was based on a predefined UPDRS motor score difference between the immediate release and prolonged release groups of at least three points on the UPDRS motor subscale. Therefore, ropinirole prolonged release was concluded to be non-inferior to ropinirole immediate release. There were no significant differences between groups in UPDRS activities of daily living (ADL), Beck Depression Inventory scores, Epworth Sleepiness Scale scores or Parkinson's Disease Sleep Scale scores. It is important to mention that overall medication compliance was significantly better in those taking ropinirole prolonged release (97\%) compared to ropinirole immediate release $(88 \%-92 \% ; \mathrm{p}<0.01)$.

The authors concluded that ropinirole prolonged release was not inferior to ropinirole immediate release and that it was a safe and effective treatment for early PD. They also demonstrated that an overnight switch with an approximate 1:1 conversion ratio from ropinirole immediate release to ropinirole prolonged release was well tolerated.

\section{Advanced Parkinson's disease}

The EASE-PD Adjunct study was a multicenter, randomized, double-blinded, placebo-controlled trial that examined the use of once-daily ropinirole prolonged release or placebo for 24 weeks in 393 patients with idiopathic PD inadequately controlled by levodopa. ${ }^{32}$ Participants were required to be within Hoehn and Yahr stage II-IV, to be on a stable dose of levodopa for the preceding 4 weeks and to have at least 3 hours of daily "off" time during normal waking hours. Patients were also allowed to take amantadine, anticholinergic medications, COMT inhibitors and/or selegiline as long as no changes in dosing were made within 4 weeks prior to study participation.

All participants received placebo for 14 days and were then randomized to receive either once-daily ropinirole prolonged release (202 subjects) or to continue taking placebo (191 subjects). ${ }^{32}$ Initial study medication doses of $2 \mathrm{mg}$ were 
gradually titrated to an effective therapeutic response or the development of adverse effects. All patients were titrated to a minimum of $6 \mathrm{mg}$ /day and $50 \%$ were maintained at the study's maximum dose of $24 \mathrm{mg} /$ day. There was a mandatory reduction in levodopa dose once patients reached a daily study medication dose of $8 \mathrm{mg}$. The primary endpoint was the mean change from baseline at week 24 in overall awake time spent "off" as measured by reviewing patient diaries. The mean final dose of ropinirole prolonged release was $18.8 \mathrm{mg}$ /day. Ropinirole prolonged release significantly decreased the average "off" awake time by 2.1 hours daily compared to 0.3 hours daily by placebo, which results in an adjusted treatment effect of 1.7 fewer "off” hours a day with ropinirole prolonged release. UPDRS motor scores were improved by 6.5 points with ropinirole prolonged release compared to 1.7 points with placebo $(\mathrm{p}<0.0001)$.

The study allowed levodopa doses to be reduced based on each individual's clinical improvement as is commonly encountered among patients on combination therapy with levodopa and a dopamine agonist. At week 24, dose reduction in levodopa was more common in those receiving ropinirole prolonged release $(278 \mathrm{mg}$ /day reduction) therapy than placebo (164 mg/day reduction). ${ }^{32}$ Of note, a significant reduction in "off" time was noted as early as week two using a $4 \mathrm{mg}$ daily dose of ropinirole prolonged release. More subjects taking active drug were considered to be "very much improved" or "much improved" as rated by the Clinical Global Impression-Improvement (CGI-I) scale by week 24 than placebo ( $42 \%$ vs $14 \%){ }^{32}$

Several significant secondary endpoints were noted including significant improvements in ADL as measured by the UPDRS ADL score (3.5 points for ropinirole prolonged release vs 0.9 points for placebo; $p<0.0001$ ) and a significant reduction in depression as assessed by the Beck Depression Inventory II (BDI-II) scale (2.1 points for ropinirole prolonged release vs 0.5 points for placebo; $\mathrm{p}<0.01$ ). There were also significant improvements in several aspects of quality of life as measured by the Parkinson's Disease Quality of Life Questionnaire-39 (PDQ-39); These included the subscores of mobility ( $p<0.0001)$, activities of daily living $(\mathrm{p}<0.0001)$, emotional well-being ( $\mathrm{p}<0.015)$, stigma $(\mathrm{p}<0.02)$, and communication $(\mathrm{p}<0.02) .{ }^{32}$ There were no significant changes in PDQ-39 subscales of social support, cognition or bodily discomfort.

Ropinirole prolonged release was demonstrated to be a safe and effective treatment as an adjunct to levodopa for patients with advanced PD which allowed for a significant reduction in daily "off" time and a reduction in daily levodopa dose.
In addition, it was demonstrated that ropinirole prolonged release not only improved the motor symptoms of PD compared to placebo but also the non-motor symptoms of the disease such as depression and quality of life. ${ }^{32}$

\section{Adverse effects}

In the early PD study, adverse effects were similar between ropinirole prolonged and immediate release formulations. The adverse events that occurred in greater than $5 \%$ of subjects in either treatment group included nausea (19\% prolonged release, $20 \%$ immediate release), somnolence ( $11 \%$ prolonged release, $15 \%$ immediate release), dizziness ( $6 \%$ prolonged and immediate release), headache ( $6 \%$ prolonged release, $5 \%$ immediate release), constipation ( $5 \%$ prolonged and immediate release), dyspepsia (3\% prolonged release, $7 \%$ immediate release) and fatigue ( $3 \%$ prolonged release, $5 \%$ immediate release). ${ }^{31}$

In the advanced PD study, the most common adverse effects noted with ropinirole prolonged release versus placebo included the development of dyskinesia (13\% vs 3\%), nausea ( $11 \%$ vs $4 \%$ ), dizziness ( $8 \%$ vs $3 \%$ ), somnolence ( $7 \%$ vs $4 \%$ ), hallucinations ( $6 \%$ vs $1 \%$ ), and orthostatic hypotension ( $5 \%$ vs $2 \%) .{ }^{32}$ Withdrawal rates from the EASE-PD Adjunct study were comparable between ropinirole prolonged release and placebo, with $5 \%$ of each group withdrawing from the study. Although ropinirole may worsen pre-existing dyskinesia or increase the development of dyskinesia in patients with $\mathrm{PD}$, dyskinesia can often be managed by decreasing the concomitant dose of levodopa. It is worth noting that $48 \%$ of the 27 advanced PD trial participants who reported dyskinesia at any time during the study had already reported their presence at baseline. ${ }^{32}$ No significant changes in supine blood pressure were noted in subjects participating in clinical trials. ${ }^{29}$

\section{Conclusion}

There are several potential advantages in using ropinirole prolonged release versus the immediate release formulation, especially during the more advanced stages of PD. Many patients with motor fluctuations cycle between "on" and "off" states so maintaining more consistent dopaminergic activity using ropinirole prolonged release could alleviate or potentially even delay the onset of these fluctuations. Furthermore, slower absorption and steadier plasma levels with ropinirole prolonged release are likely to increase tolerability by reducing adverse effects associated with the more marked peak and troughs of the immediate release formulation. In addition, the simpler titration schedule may allow the patient to achieve clinically desired results more quickly than with the immediate release formulation. ${ }^{31}$ Once-daily instead of multiple divided doses is 
also likely to increase patient compliance as most patients with PD are already on a complicated regimen.

Ropinirole prolonged release appears to be a safe, well-tolerated option for treating early and advanced PD. This medication has a wide spectrum of use in PD and can include patients who need initial monotherapy, those insufficiently controlled by levodopa, and those already taking a dopamine agonist with motor fluctuations related to variations in plasma levels. Patients who are prescribed this medication are more likely to witness objective improvement in motor function as well as subjective improvement in ADL, several important aspects regarding quality of life, and possibly even depression. Several pending studies will likely elaborate more on comparisons between immediate and prolonged release forms of ropinirole although the adverse effect profiles appear to be similar. Because immediate release ropinirole is approved for restless legs syndrome, further studies using ropinirole prolonged release in restless legs syndrome are also likely in the future.

\section{Disclosures}

Dr. Pahwa has served as a consultant for and received honoraria from GlaxoSmithKline, Boehringer Ingelheim, Impax, Teva Neuroscience, Medtronic, Novartis and Valeant.

Dr. Lyons has served as a consultant for and received honoraria from GlaxoSmithKline, Teva Neuroscience, Novartis, Medtronic and St. Jude Medical.

\section{References}

1. Strickland D, Bertoni JM. Parkinson's prevalence estimated by a state registry. Mov Disord. 2004;19:318-323.

2. Twelves D, Perkins KS, Counsell C. Systematic review of incidence studies of Parkinson's disease. Mov Disord. 2003;18:19-31.

3. Elbaz A, Bower JH, Maraganore DM, et al. Risk tables for parkinsonism and Parkinson's disease. J Clin Epidemiol. 2002;55:25-31.

4. Miyasaki JM, Martin W, Suchowersky O, Weiner WJ, Lang AE. Practice parameter: initiation of treatment for Parkinson's disease: an evidence-based review: report of the Quality Standards Subcommittee of the American Academy of Neurology. Neurology. 2002;58:11-17.

5. Goetz CG, Poewe W, Rascol O, Sampaio C. Evidence-based medical review update: pharmacological and surgical treatments of Parkinson's disease: 2001 to 2004. Mov Disord. 2005;20:523-539.

6. Horstink M, Tolosa E, Bonuccelli U, et al. Review of the therapeutic management of Parkinson's disease. Report of a joint task force of the European Federation of Neurological Societies and the Movement Disorder Society-European Section. Part I: early (uncomplicated) Parkinson's disease. Eur J Neurol. 2006;13:1170-1185.

7. Pahwa R, Factor SA, Lyons KE, et al. Practice Parameter: treatment of Parkinson disease with motor fluctuations and dyskinesia (an evidencebased review): report of the Quality Standards Subcommittee of the American Academy of Neurology. Neurology. 2006;66:983-995.

8. Olanow CW, Watts RL, Koller WC. An algorithm (decision tree) for the management of Parkinson's disease (2001): treatment guidelines. Neurology. 2001;56:S1-S88.

9. Parkinson Study Group. Levodopa and the progression of Parkinson's disease. N Engl J Med. 2004;351:2498-2508.
10. Obeso JA, Rodriguez-Oroz MC, Chana P, Lera G, Rodriguez M, Olanow CW. The evolution and origin of motor complications in Parkinson's disease. Neurology. 2000;55:S13-S20; discussion S21-S13.

11. Schrag A, Quinn N. Dyskinesias and motor fluctuations in Parkinson's disease. A community-based study. Brain. 2000;123( Pt 11):2297-2305.

12. Sharma JC, Ross IN, Rascol O, Brooks D. Relationship between weight, levodopa and dyskinesia: the significance of levodopa dose per kilogram body weight. Eur J Neurol. 2008;15:493-496.

13. Olanow CW, Rascol O, ADAGIO Investigators. ADAGIO: A prospective, double-blind, delayed-start study to examine the potential disease modifying effect of rasagiline in early Parkinson's disease. Mov Disord. 2008:S194.

14. Rascol O, Brooks DJ, Korczyn AD, De Deyn PP, Clarke CE, Lang AE. A five-year study of the incidence of dyskinesia in patients with early Parkinson's disease who were treated with ropinirole or levodopa. 056 Study Group. N Engl J Med. 2000;342:1484-1491.

15. Parkinson Study Group. Pramipexole vs levodopa as initial treatment for Parkinson disease: a 4-year randomized controlled trial. Arch Neurol. 2004;61:1044-1053.

16. Mamikonyan E, Siderowf AD, Duda JE, et al. Long-term follow-up of impulse control disorders in Parkinson's disease. Mov Disord. 2008;23:75-80.

17. Pham DQ, Nogid A. Rotigotine transdermal system for the treatment of Parkinson's disease. Clin Ther. 2008;30:813-824.

18. Antonini A, Poewe W. Fibrotic heart-valve reactions to dopamine-agonist treatment in Parkinson's disease. Lancet Neurol. 2007;6:826-829.

19. Zanettini R, Antonini A, Gatto G, Gentile R, Tesei S, Pezzoli G. Valvular heart disease and the use of dopamine agonists for Parkinson's disease. N Engl J Med. 2007;356:39-46.

20. Snow BJ, Macdonald L, McAuley D, Wallis W. The effect of amantadine on levodopa-induced dyskinesias in Parkinson's disease: a double-blind, placebo-controlled study. Clin Neuropharmacol. 2000;23:82-85.

21. Del Dotto P, Pavese N, Gambaccini G, et al. Intravenous amantadine improves levadopa-induced dyskinesias: an acute double-blind placebocontrolled study. Mov Disord. 2001;16:515-520.

22. Waters CH, Sethi KD, Hauser RA, Molho E, Bertoni JM. Zydis selegiline reduces off time in Parkinson's disease patients with motor fluctuations: a 3-month, randomized, placebo-controlled study. Mov Disord. 2004;19:426-432.

23. LeWitt PA, Lyons KE, Pahwa R. Advanced Parkinson disease treated with rotigotine transdermal system: PREFER Study. Neurology. 2007;68:1262-1267.

24. Dewey RB Jr, Hutton JT, LeWitt PA, Factor SA. A randomized, doubleblind, placebo-controlled trial of subcutaneously injected apomorphine for parkinsonian off-state events. Arch Neurol. 2001;58:1385-1392.

25. Leopold NA, Polansky M, Hurka MR. Drug adherence in Parkinson's disease. Mov Disord. 2004;19:513-517.

26. Grosset D, Antonini A, Canesi M, et al. Adherence to antiparkinson medication in a multicenter European study. Mov Disord. 2009. Feb 3 [Epub ahead of print].

27. Requip (ropinirole hydrochloride): US Prescribing Information: GlaxoSmithKline; 2006.

28. Kaye CM, Nicholls B. Clinical pharmacokinetics of ropinirole. Clin Pharmacokinet. 2000;39:243-254.

29. Requip XL (ropinirole extended release tablets): US Prescribing Information: GlaxoSmithKline; 2008.

30. Tompson DJ, Vearer D. Steady-state pharmacokinetic properties of a 24-hour prolonged-release formulation of ropinirole: Results of two randomized studies in patients with Parkinson's disease. Clin Ther. 2007;29:2654-2666.

31. Stocchi F, Hersh BP, Scott BL, Nausieda PA, Giorgi L. Ropinirole 24-hour prolonged release and ropinirole immediate release in early Parkinson's disease: a randomized, double-blind, non-inferiority crossover study. Curr Med Res Opin. 2008;24:2883-2895.

32. Pahwa R, Stacy MA, Factor SA, et al. Ropinirole 24-hour prolonged release: randomized, controlled study in advanced Parkinson disease. Neurology. 2007;68:1108-1115. 